\title{
LA VINCULACIÓN DE LA EDUCACIÓN Y GÉNERO
} LINKING EDUCATION AND GENDER

\author{
Volumen 13, Número 1 \\ Enero - Abril \\ pp. 1-16
}

Este número se publicó el 30 de enero de 2013

Mario Castillo Sánchez
Ronny Gamboa Araya

Revista indizada en REDALYC, SCIELO

Revista distribuida en las bases de datos:

CATÁLOGO DE LATINDEX, IRESIE, CLASE, DIALNET, DOAJ, E-REVIST@S, SHERPA/ROMEO, QUALIS

Revista registrada en los directorios:

ULRICH'S, $\underline{\text { REDIE}}, \underline{\text { RINACE}}, \underline{\text { OEI }}$ MAESTROTECA, PREAL, $\underline{\text { CLASCO }}$ 


\title{
LA VINCULACIÓN DE LA EDUCACIÓN Y GÉNERO LINKING EDUCATION AND GENDER
}

\author{
Mario Castillo Sánchez 1 \\ Ronny Gamboa Araya ${ }^{2}$
}

\begin{abstract}
Resumen: Este ensayo pretende abordar la vinculación existente entre el papel de la educación y el rol del género a través del abordaje de temas como las relaciones sexistas en la educación, formas de discriminación y transversalización de género en la educación. En la educación actual hemos normalizado la desigualdad, lo que ha influido en que tanto hombres como mujeres adopten estos roles consciente o inconscientemente. Por lo tanto, es innegable el papel que la educación ha tenido en la reproducción de los modelos de desigualdad y jerarquización entre hombres y mujeres. Aunque se haya legislado a favor de la equidad de género, en el uso del lenguaje inclusivo, en las cuotas de participación en organismos políticos, en la representación para diferentes instituciones, la creación de entes encargados de velar por un trato igualitario entre hombres y mujeres, estos esfuerzos resultan insuficientes. La igualdad de género requiere un proceso de aculturación que cambie concepciones y prácticas socialmente aprendidas. Por tal razón, es necesario un cambio orientado hacia la igualdad en la práctica educativa que incluya a todas las personas involucradas y permita desarrollar una educación sin discriminación.
\end{abstract}

Palabras clave: EDUCACIÓN, EQUIDAD DE GÉNERO, DESIGUALDAD, SEXISMO.

Abstract: This paper pretends to address the relation between the role of education and the role of gender by addressing topics such as sexist-content of education, forms of discrimination, and gender mainstreaming in education. In today's education we have normalized inequality in such a way that both, men and women, adopt these roles consciously or unconsciously. Therefore, it is undeniable the role that education has in reproducing patterns of inequality and hierarchy between men and women. Although it has been legislated in favor of gender equity, in the use of inclusive language, in the participation quotas in political aspects, representing different institutions, the creation of bodies responsible for ensuring equal treatment between men and women still insufficient because gender equality requires a process of acculturation to change socially learned concepts and practices. For this reason, we need a oriented change toward gender equality in educational practice that allows educational development without discrimination and including all people involved.

Key words: EDUCATION, GENDER EQUITY, INEQUALITY, SEXISM.

\footnotetext{
1 Docente de la Escuela de Matemática de la Universidad Nacional y de la Escuela de Salud Pública de la Universidad de Costa Rica. Doctor en Educación por la Universidad Estatal a Distancia, Costa Rica. Máster en Estadística por la Universidad de Costa Rica. Dirección electrónica: mario.castillo.sanchez.@una.cr

2 Investigador y académico de la Escuela de Matemática de la Universidad Nacional, Costa Rica. Máster en Matemática Educativa por el Centro de Investigación y Estudios Avanzados del Instituto Politécnico Nacional, México. Licenciado en la Enseñanza de la Matemática por la Universidad Nacional. Dirección electrónica: ronny.gamboa.araya@una.cr
}

Ensayo recibido: 21 de agosto, 2012

Aprobado: 22 de noviembre, 2012 


\section{Introducción}

Debido a que la escuela recibe a hombres y mujeres socializados en géneros masculino y femenino, esta puede jugar dos papeles: ayudar a reforzar, mantener, producir y reproducir los estereotipos de género (Tomé, 1999; Graña, 2008), o, ayudar a modificarlos y colaborar con su desconstrucción (Subirats y Brullet, 1999).

Género es una categoría que permite analizar las relaciones sociales entre hombres y mujeres. Por género se entiende la construcción social de los sentidos que para las sociedades tiene el ser hombre o mujer, significados que condicionan la actuación, valoración y distribución del poder (real y simbólico) inequitativo para las mujeres como grupo. (González, 2003, pp. 132-133)

El proceso de socialización que se da en los primeros años escolares contribuye a la formación de la identidad social, específicamente el rol género. El papel de la educación es fundamental para visibilizar las desigualdades existentes en la sociedad, muchas de las cuales se han considerado parte de ella.

Este ensayo pretender abordar la vinculación existente entre el papel de la educación y el rol del género a través del abordaje de temas como los contenidos sexistas de la educación, formas de discriminación, transversalización de género en la educación y formación docente y coeducación.

\section{Las relaciones sexistas en la educación}

Subirats y Brullet (1999) señalan que dado que se ha considerado que la escuela es la institución encargada de formar al ciudadano, el paso por esta ha sido y es obligatorio. abordaje que el sistema educativo brinda a las diferencias de sexo depende de las complejas relaciones que se establecen en el orden patriarcal y social dominante en cada momento histórico.

Por lo anterior, es imposible separar la educación del contexto social e histórico en el cual se encuentra inmersa, pues representa un fenómeno social que posee una dimensión social y política que le otorga complejidad a los hechos y las relaciones educativas (Araya, 2004).

Los estudios de género han puesto en evidencia patrones sexistas en la socialización escolar que reproducen comportamientos, expectativas y opciones asociadas al género 
(Graña, 2008). Araya (2004) indica que fue desde la pedagogía crítica donde se empezó a concebir el sistema educativo como un espacio investido de poder, no neutral, por lo que contribuía a crear y legitimar identidades socialmente jerarquizadas.

En efecto, "el sexismo es una forma de discriminación que utiliza al sexo como criterio de atribución de capacidades, valoraciones y significados creados en la vida social" (Araya, 2004, p. 1). Al respecto, Subirats (1994) señala que el término sexismo se utiliza, en Ciencias Sociales, para designar aquellas actitudes que introducen desigualdad y la jerarquización en el trato que reciben los individuos, sobre la base de diferenciación del sexo. El sexismo provoca consecuencias negativas para todos los individuos, pues limita sus posibilidades como persona y les niega determinados comportamientos, duplicándose estas para las mujeres, pues la sitúa en una posición de inferioridad y de dependencia.

La ordenación educativa sexista interviene en la construcción de la personalidad de los individuos y determinan una internalización de normas de género diferenciadas, que incluyen distintas expectativas y posibilidades, fuerza de trabajo y jerarquización de los individuos (Subirats y Brullet, 1999).

Por su parte, Tomé (1999) señala que el sexismo es un problema que afecta tanto a las mujeres como a los hombres. Representa, además, un problema de desigualdad social, donde las mujeres ven limitadas sus oportunidades de ingreso a ciertas funciones sociales, y un problema de jerarquía cultural, ya que tanto la escuela como la sociedad realizan valoraciones asociadas a la masculinidad, despreciando la identidad femenina.

En el currículo explícito el sexismo se refleja en las determinaciones de las políticas acerca de lo que se enseña y no se enseña; el lenguaje, que contiene un uso regular y normativo del masculino para designar personas de ambos sexos; y en los textos escolares, que por excelencia transmiten papeles tradicionales asignados a hombres y mujeres (Araya, 2004).

Subirats y Brullet (1999) mencionan que desde el nacimiento de la escuela moderna se postulaba que los niños y niñas debían ser educados de manera distinta. Incluso citan a Rousseau, quien había dicho que la niña debía ser educada como ser dependiente y el niño como ser autónomo. Así, mencionan que la educación no solo es utilizada para legitimar la desigualdad, haciéndola parecer como resultado de las características individuales, sino que debía formar individuos con características diferentes, lo que concuerda con las exigencias 
de una sociedad con una marcada división del trabajo; es por ello que surge la necesidad de "producir" individuos con una fuerza de trabajo diferenciada y jerarquizada.

En un inicio, la educación de las mujeres giraba en torno a los rezos, el aprendizaje doméstico y la exclusión de algunas materias descritas para los hombres. Se argumentaba que las niñas no debían estudiar ni necesitaban una cultura profunda, pues ello las podía alejar de su función principal: la de esposas y madres (Subirats, 1994).

Subirats (1994) señala que en la educación de los individuos se ha considerado como importante para la vida adulta materias como matemáticas, historia o lenguaje. Sin embargo, no se ha considerado como necesario aprender a cuidar un recién nacido, preparar comida, conocer los efectos del lavado en la ropa, entre otros. Esto debido, principalmente, a que no se les considera como un saber fundamental y han sido relegados como parte de los conocimientos "femeninos".

Graña (2008) señala que a los hombres se les educa para manejar el poder, mientras que las mujeres son instruidas para que impriman, en las distintas actividades que desarrollen, los roles maternal y domésticos.

A los hombres se les ha educado para que orienten la actividad científica (Graña, 2008). Por ello, señala el autor, a las mujeres se les ha orientado hacia carreras de las áreas de letras y humanidades, como Odontología, Ciencias Sociales, Medicina, Psicología. Los varones dominan en áreas como las Ingenierías, Arquitectura, Matemáticas, Derecho y Ciencias Naturales.

En ese sentido, Graña (2008) señala que la escolaridad de las mujeres ha sido más fluida que la de los varones (ellas repiten menos); los hombres han presentado mejor rendimiento en matemáticas, ciencias y técnicas, mientras que las mujeres se han desempeñado mejor en lenguas; a las mujeres se les desestimula para seguir ciertas carreras aunque inicialmente expresen deseos de cursarlas, por lo que al finalizar la secundaria muchas de ellas se inclinan por carreras "razonablemente femeninas" (Graña, 2008).

Al respecto, Acker (2003) puntualiza que una explicación común para dar cuenta del fracaso de las mujeres para alcanzar una posición "privilegiada" es la que otorga la responsabilidad a los padres, a las escuelas y otros agentes socializadores que ha hecho que la mujer, desde su infancia, desarrolle una serie de características que no facilitan el éxito, principalmente en campos tradicionalmente dominados por hombres. 
Por eso, la educación, de forma explícita o implícita, se ha constituido como un medio para la reproducción de los esquemas sociales de desigualdad y la perpetuación de las identidades de género socialmente determinadas.

\section{La expresión de las distintas formas de discriminación}

La discriminación para las mujeres se ha dado de diversas formas. Por ejemplo, históricamente la ciencia está explicada, en su mayor parte, por hombres; tanto así, que a las mujeres se les ha excluido del reconocimiento sobre los aportes culturales y científicos realizados a la humanidad que subvalora el trabajo realizado por ellas (Subirats, 1994).

Fioretti, Tejero y Díaz (2002) señalan que históricamente a las mujeres no se les ha reconocido que parte de la producción social se ha dado a través de ellas, pues los valores patriarcales que han dominado han logrado ordenar el registro histórico para presentar las actividades de los hombres como más importantes para las sociedades, lo que las ha llevado a aparecer como contribuyentes "marginales" del desarrollo humano.

También, se ha producido discriminación en el tipo de investigación que se ha realizado. Acker (2003) señala, basada en los resultados reportados por varios artículos de investigación en sociología y educación, que pareciera que la cantidad de hombres que van a la escuela es mucho mayor que el de las mujeres, que no hay mujeres influyentes; que las mujeres no pasan de "la escuela al trabajo"; que nunca asisten a los centros educativos para recibir una mayor formación, y que prácticamente no hay mujeres adultas en el mercado laboral, excepto por algunas profesoras, trabajadoras sociales y enfermeras. Sin embargo, consideramos en la actualidad esta situación ha cambiado, pues la participación de las mujeres en el sector productivo de la población ha aumentado, así como en la representación política. Por ejemplo, en Costa Rica cerca del 39 \% de la integración del Poder Legislativo corresponde a mujeres.

La autora argumenta que se ha excluido a las mujeres de estudios como la transición de la escuela al trabajo, aspiraciones laborales, autoconcepto profesional u ocupacional; en otros casos, las muestras de los distintos estudios han sido hombres propiamente, excluyendo de esta forma los análisis sobre la experiencia de las mujeres. Sobre lo anterior, Acker (2003) señala que ello refleja la representación desproporcionada de los hombres entre las "élites sociales", la suposición de que el trabajo de los hombres es de mayor 
importancia e interés que el de las mujeres y a que muchos de los fenómenos estudiados por los varones se inclinan hacia versiones masculinas.

Otro aspecto de la investigación es que muchos autores no siempre analizan datos a partir de las diferencias sexuales, aún cuando ello pudiese haber sido relevante, y cuando lo han realizado parecen no saber cómo interpretar sus resultados (Acker, 2003). Pareciera, entonces, que la investigación sobre mujeres ha sido "poco relevante". La tendencia ha sido "una exclusión de las chicas de las muestras o una conceptualización de sus opciones, simplemente entre matrimonio o carrera" (Acker, 2003, p. 57)

El lenguaje ha representado otra forma de discriminación (Subirats, 1994). Acker (2003) menciona, por ejemplo, que en la revistas ha sido convencional el uso de "él" para referirse a "él y ella" u "hombre" por "humanidad", por lo que se ha dado la idea de que las mujeres literalmente no existen o no son relevantes para el tema en cuestión. Igualmente, el lenguaje utilizado para referirse a las profesiones u ocupaciones denotan discriminación de género ya sea que dicha profesión implique un grado de prestigio y poder o subordinación. Por ejemplo, presidente, gerente, médico, sirvienta, ama de casa, maestra, entre otros.

Una forma de discriminación señalada por Belmonte y Guillamón (2008) es la tipificación de los "papeles" femeninos y masculinos que distintos programas de televisión realizan, contribuyendo a la jerarquización de la división del trabajo y la transmisión de estereotipos de género, tanto en el ámbito laboral o público como personal o privado, que actúan como modelos de desigualdad y representan una fuente de educación informal por medio de la televisión.

La historia de la educación de las mujeres muestra cómo a lo largo del tiempo estas han ocupado un lugar secundario y subordinado (Subirats, 1994). Subirats y Brullet (1999) señalan que en educación existe una tendencia de las y los docentes a prestar mayor atención a los hombres (en casi todos los aspectos), discriminar lingüísticamente entre ambos sexos y señalar comportamientos diferentes entre ellos y ellas. A los varones se les da mayor atención, porque se ha partido del hecho de que presentan más problemas de disciplina; a ellas se les da menos la palabra dentro de la clase, porque son más discretas (Graña, 2008).

Respecto al sexismo en la profesión educativa, se puede decir que la enseñanza es uno de los sectores más feminizados; sin embargo, las posiciones de las mujeres en la estructura educativa suelen ser muy inferiores a los hombres (Subirats, 1994). 
Acker (2003) señala algunas diferencias entre mujeres y hombres y su papel en la educación. Aunque casi la totalidad del profesorado y dirección de las guarderías y escuelas están ocupados por mujeres, el número de estas disminuye conforme se avanza en los estudios o niveles; además, la mayoría del profesorado a tiempo parcial son mujeres. Una de las consecuencias de esta situación es, como lo indica la autora, la diferencia en los salarios.

Acker (2003) señala una diferencia ente las funciones establecidas para los y las docentes. Esta diferenciación radica en las asignaturas que se enseñan y en las responsabilidades asignadas. Así, a las mujeres se les ha "conferido" la enseñanza a niños y niñas pequeñas y a grupos de su mismo sexo, principalmente desempeñándose en asignaturas domésticas y humanidades; a los hombres se les ha delegado la enseñanza de los varones y se han desarrollado en materias tecnológicas, administrativas y curriculares. Estas consideraciones, según Acker (2003), permiten hablar de una división sexual del trabajo en la enseñanza. Aunque esta situación ha variado paulatinamente aún cuando en educación primaria el personal docente sea, en su mayoría, mujeres, mientras que en secundaria dominan los hombres.

Debido a que la enseñanza ha estado asociada con las mujeres, esto provocó que durante algún tiempo haya sido considerada como una semi-profesión y no goce del prestigio dado a las tradicionalmente "dominadas" por los hombres.

\section{La transversalización de género en la educación}

Tomé (1999) y Graña (2008) indican que la escuela recibe a hombres y mujeres socializados en géneros masculino y femenino, por lo que, al no intervenir, refuerza, mantiene, produce y reproduce los estereotipos de género. Aunado a lo anterior, señalan que en muchas ocasiones se trata de un problema que ni el profesorado ni el alumnado son conscientes de que se dé.

Por tal motivo, Subirats y Brullet (1999) señalan que aunque el género ya ha sido adquirido, en parte, al entrar a la escuela, esta puede reforzar su construcción, modificarla o incluso colaborar con su desconstrucción. El género es un principio organizador "mayor" en la educación que se aplica a uniformes, asignaturas curriculares, prácticas administrativas, actividades de clase y uso del espacio en el aula y fuera de ella (Acker, 2003). Tanto es su influencia que, según la autora, es sorprendente la forma en que las escuelas mixtas han 
usado el sexo como un clasificador administrativo para organizar los registros, organización del mobiliario en las aulas, para hacer filas, entre otros. La autora apunta que el análisis de los regímenes de género, códigos de género o currículo oculto no pueden culpar al profesorado, pues su acción parece afianzada en la tradición y en la rutina, por lo que se requiere tomar acciones concretas para corregir dichas prácticas

A los hombres aún se les educa para ejercer la violencia (como si fuera una habilidad necesaria y cotidiana), mientras que a las mujeres se les enseña en atención a la belleza, como si su futuro continuara dependiendo de sus posibilidades en el mercado del matrimonio (Subirats, 1999).

De hecho, Acker (2003) menciona que la actividad feminista se ha encauzado hacia los procesos sociales dentro del aula y ha comenzado a percibirse que las formas en la escolarización contribuyen a la reproducción de las relaciones de género. Así, el sexo ha funcionado como un argumento para la diferenciación a través de la vida escolar, donde el alumnado se vuelve hombre o mujer por inscripción, lugar donde se sientan, el trato, entre otros. Esto ha provocado que el profesorado se dirija a ellos y ellas como tales, les pongan a competir y les evalúe y sitúe en lo que equivale a una educación no mixta dentro de una educación mixta (mayoritariamente).

Es más, Acker (2003) indica que muchos de los estudios sobre género y educación sostienen que el profesorado posee un papel importante en la frustración del potencial de las niñas, ya sea mediante el trato y las expectativas diferenciales de cada sexo o como parte del régimen de género de la escuela, por medio de los modelos de masculinidad y feminidad contenidas en las prácticas cotidianas como agrupación del alumnado, horarios y en la división sexual del trabajo del profesorado. La mayoría de los estudios sugieren que los hombres obtienen más atención por el profesado, independiente del sexo, y que los contenidos y trabajo en el aula se planifican, frecuentemente, en función de ellos (Tomé, 1999; Acker, 2003).

Por su parte, Subirats (1999) indica que fue en los años setenta cuando se comenzó a analizar el sistema educativo como no neutral, un escenario donde los grupos sociales se enfrentaban y luchaban por los recursos y por el poder, donde cada grupo trataba de maximizar, en el sistema educativo, sus posibilidades, por medio del control de la cultura y de las reglas que determinaban el funcionamiento de las distintas instituciones sociales. Según esta autora, la influencia política de los grupos sociales con más poder son los que 
marcan más profundamente las normas escolares, de modo que, aún sin preverlo, la escuela se convierte en un medio de reproducción del sistema social existente y de las posiciones de poder y de jerarquía vigentes en la sociedad.

La escuela, el aula, la interacción cotidiana, permitieron descubrir que el sistema educativo es mucho más que un expendedor de títulos, es un espacio de socialización diferenciada, en el que hay reglas sumamente estrictas, aunque invisibles, que moldean con gran precisión las personalidades individuales, que construyen el éxito y el fracaso, que separan quienes están destinados a tener responsabilidades y a tomar decisiones de quienes están destinados a plegarse a ellas. (Subirats, 1999, pp. 21-22)

Además, Subirats (1999) señala que las mujeres y hombres no se constituyen como tales solamente en función del sexo, pues la sociedad ha creado sistema de roles y patrones de comportamiento distintos a cada uno de ellos, no se puede atribuir solamente al sistema educativo la responsabilidad moral de la socialización genérica (aunque muchos de los intentos de cambio hayan comenzado en el ámbito educativo), pues tanto la familia como el entorno inmediato y las instituciones e instancias sociales desempeñan un importante papel en ello.

Sin embargo, en la medida en que las diferencias pueden considerarse producidas por el efecto socializador llevado a cabo por las instituciones educativas, se ha producido una revisión de las características curriculares, de los libros de texto, variables conceptuales, del lenguaje, currículo oculto y, en general, de la toda la práctica educativa para determinar en qué medida esta está regida por una ideología sexista y qué consecuencias tiene para las mujeres (Subirats, 1999).

La autora señala que los hallazgos han permitido determinar que los currículos son sexistas, los libros de texto ignoran la presencia de las mujeres y cuando lo hacen es en papeles tradicionales, que el lenguaje utilizado por la escuela invisibiliza a las mujeres y se da mayor participación a los hombres en las distintas actividades. Algunos estudios han señalado que el espacio (tanto dentro como fuera del aula) ha sido divido en "territorio de ellos" y "territorio de ellas" (Subirats, 1999; Tomé, 1999; Acker, 2003).

Según, Subirats (1999) y Subirats y Brullet (1999), dado que el sistema educativo ha sido diseñado desde los hombres y para los hombres, el fin es la reproducción de la masculinidad. Debido a ello, las mujeres continúan siendo figuras secundarias. Como 
ninguno de los comportamientos femeninos concuerdan con el sistema educativo, muy pocos de estos son transmitidos por la escuela, y los que sí han sido contemplados se transfieren en la forma de un valor subordinado. Así, aunque destaquen académicamente no podrán tener las mismas oportunidades, por lo que realizan el aprendizaje de la subordinación sin combatirla, aceptándola.

Esta valoración diferencial entre hombres y mujeres, por lo general sutil y encubierta, establecen un orden simbólico relacionado con el poder, que ha señalado lo que es legítimo para cada grupo sexual, contribuyendo a la perpetuación de los géneros (Subirats, 1999).

Tomé (1999) señala que la producción de diversos estudios puso en evidencia la permanencia de un conjunto de normas, explícitas e implícitas, que mantienen y reproducen las formas de discriminación en el sistema educativo. Realizar las acciones necesarias para superar las formas de discriminación es el reto actual que se le presenta a la educación.

\section{La formación docente y la coeducación}

Tomé (1999) señala que el sexismo educativo responde a una forma rutinaria de transmisión ideológica en la que el profesorado desconoce el sistema de valores que transmite y las consecuencias de su propia actuación en dicho ámbito. Acker (2003) indica que uno de los aspectos más criticados en algunos estudios es la insistencia en que las aspiraciones de las mujeres y las responsabilidades del papel familiar son suficientes para explicar su posición subordinada en la enseñanza.

Asimismo, Acker (2003) menciona que la división sexual del trabajo entre el profesorado contribuye a la reproducción del orden social patriarcal y/o capitalista; ello mediante la reproducción de modelos de relaciones de hombre-mujer y responsabilidades sexualmente diferenciadas.

Señala que aunque el profesorado está comprometido con el avance de los intereses de todos los sujetos a su cargo, las iniciativas sobre la igualdad de género son raras y no consiguen transformar sus actitudes y acciones, debido tanto a la naturaleza de las iniciativas como a las características, ideas y condiciones de trabajo del profesorado. Este se resiste a la iniciativas anti-sexistas, en la mayoría de los casos, debido a que no reconocen que exista tal problema educativo. Aunque el discurso feminista está al alcance de todo y toda docente, no resulta familiar ni cómodo y tiene como posible consecuencia el alejamiento de los colegas (Acker, 2003). 
Las anécdotas influidas por el género del propio profesorado, los roles fuera de la escuela o institución educativa y las interacciones dentro de la misma moldean sus creencias sobre lo posible o deseable para reformar; sin embargo, actualmente las profesoras parecen más sensibilizadas en sus carreras que en el pasado y los problemas de género se nombran, poco a poco, en los cursos de formación del profesorado (Acker, 2003).

Respecto al profesorado, Acker (2003) menciona que las investigaciones han señalado que las profesoras, por lo general, se encuentran divididas entre los compromisos de la casa y el trabajo. Las experiencias sobre la familia son más importantes para las mujeres que para los hombres (Acker, 2003). Fioretti, Tejero y Díaz (2002) indican que se ha destacado, basadas en sus investigaciones, el carácter femenino de la profesión docente, ya que la docencia ha sido vista como prolongación de cierto instinto materno y de la crianza de los hijos, lo que la ha llevado a considerarse una vocación natural.

Acker (2003) señala que la ironía satura la vida de las profesoras. Tienen que vivir con las desigualdades ocasionadas por la preferencia por los hombres para las promociones y con tareas domésticas que triplican sus turnos; al mismo tiempo, les inducen a trabajar de forma constante, comprometida y dedicada. "Podría decirse que las profesoras de primaria contribuyen a la desigualdad de género con su aceptación (aún sin desearla) de esta desigualdad en sus propias vidas" (Acker, 2003, p. 154). Dentro de la cultura de su lugar de trabajo en la escuela, las profesoras, en el proceso de interpretar y percibir, se guían por sus experiencias inmediatas.

Los resultados de diversas investigaciones que pusieron de manifiesto el carácter sexista de la educación trajeron consigo una voluntad de cambio, y provocó que muchas docentes consideraran necesario cambiar la escuela y construir una educación no sexista que garantizara la igualdad de oportunidades. Pero, para ello, era necesario redescubrir a la mujer desde la didáctica y la práctica docente. Se trata, entonces, de que la escuela tenga en cuenta la existencia de los géneros, los integre en una sola cultura, los valore por igual y los transmita a toda la población escolar con independencia de su sexo (Subirats, 1999).

Subirats (1999) indica que aunque es patente la necesidad de que hombres y mujeres reciban un trato igualitario, ello no supone uniformidad, ya que las instituciones educativas se enfrentan a personas que han recibido una educación genérica, con aptitudes y necesidades diferentes. El reto está, según la autora, en la posibilidad de introducir en la escuela, en general, valores y comportamientos tanto de hombres como de mujeres y lograr que los 
pongan en práctica los individuos de los dos sexos, sin que ninguno "perjudique al otro" ni permita la implantación de jerarquías.

El modelo de escuela coeducativa, señalado por Tomé (1999), representa una forma para superar el sexismo en la educación en oposición al modelo de escuela mixta en la que existe igualdad formal en todos los aspectos pero no igualdad real.

Este modelo admite que el profesorado identifica las formas, por medio de las cuales el sexismo se manifiesta en el sistema educativo y se reconoce a sí mismo como parte de la reproducción de la desigualdad de sexos. Por lo tanto, la escuela, en general, es una institución que propicia la eliminación de las desigualdades sexuales y la jerarquía de sexos, toma en cuenta la necesidades de los grupos, reconoce la no neutralidad escolar y prepara a los individuos para el mercado laboral y para la esfera familiar y privada. De esta forma, el profesorado debe sensibilizarse y descubrir nuevas posibilidades para rehacer sus prácticas educativas y buscar soluciones propias a problemas concretos (Tomé, 1999).

Subirats (1994) señala que el término coeducación es utilizado comúnmente para referirse a la educación conjunta de dos o más grupos de población distintos. De esta forma, designa una cierta manera de entender la educación de mujeres y hombres y supone la búsqueda de mayor igualdad frente a otras opciones que promueven el mantenimiento de las diferencias.

Sin embargo, Subirats y Brullet (1999) señalan que pensar en una forma escolar coeducativa, que integre para hombres y mujeres los valores y comportamientos diferenciados de género, posee una dificultad: que aún no se sabe cuáles son los elementos positivos que se deberían rescatar del legado de las mujeres, por lo que casi siempre se termina reduciendo a las tareas domésticas. Por lo tanto, eliminar el sexismo en educación y construir una escuela coeducativa requiere instaurar una igualdad de valores y de trato entre hombres y mujeres, pero que exige rehacer el sistema de valores y actitudes que se transmiten, así como repensar los contenidos educativos.

Mientras tanto, Salomone (2007) señala argumentos dados por distintos autores a favor de un modelo de enseñanza diferenciada, donde se busca la igualdad educativa para las mujeres, la mejora del rendimiento académico global, el desarrollo del interés y la competencia en matemáticas, ciencias y tecnología, el aumento de la autoestima así como del interés y la participación en las profesiones tradicionalmente dominadas por el hombre. 
La atención se centra en vencer las desventajas sociales y educativas a las que hacen frente las mujeres.

Sin embargo, Salomone (2007) indica que los que se oponen a la enseñanza diferenciada buscan la igualdad formal e indican que esta viola el principio de que a las personas en una situación similar se les debe tratar de manera semejante. El argumento parte del hecho de que los hombres y las mujeres poseen capacidades cognitivas similares, razón por la cual no se justifica tal diferenciación. Como bien lo señala la autora, aún no hay un consenso al respecto y se debe realizar una investigación más amplia.

Para que la educación pueda contribuir con la erradicación del sexismo es necesario el reconocimiento de dicha discriminación, de la desigualdad y de la segregación como problemas educativos (Araya, 2004).

El sexismo actual puede y debe ser superado y la escuela puede contribuir a crear una sociedad en la que ni las mujeres ni los hombres vean limitadas sus posibilidades personales en función de su sexo, ni las actividades que realicen sean valoradas y medidas por la atribución a uno u otro género. (Subirats y Brullet, 1999, pp. 217-218)

Aunado a lo anterior se menciona que "Cualquier intento de cambio que no cuenta con la colaboración del profesorado tenderá al fracaso" (Tomé, 1999, p. 176). Para Araya (2004), los procesos de formación inicial del profesorado deben superar el déficit que los programas de formación presentan en el abordaje de estos temas. La educación debe formar en la diferencia y desde la diferencia para validar las características humanas como expresiones de su esencia y no de alguno de los sexos, lo cual requiere de medidas intermedias como el affidamento (relaciones de fe, fidelidad y confianza entre mujeres) que permitiría conquistas gremiales y un mayor estatus social y económico (Araya, 2004).

Subirats (1994) apunta que para alcanzar una enseñanza coeducativa hay que partir no solo de la igualdad de los individuos, sino de la integración de los modelos genéricos. Además, se debe introducir en el currículo escolar y en las relaciones de aula un conjunto de saberes que han estado ausentes, así como una mayor valoración de algunas actitudes y capacidades que han estado devaluadas. 


\section{Reflexiones finales}

No se puede negar el papel que la educación ha tenido en la reproducción de los modelos de desigualdad y jerarquización entre hombres y mujeres. Como tampoco se puede ignorar el hecho de que la educación, en general, ha servido como un medio para que los grupos dominantes implementen su ideología con el propósito de responder a sus propios intereses. En la educación actual hemos "normalizado la desigualdad" a tal punto que tanto los hombres como mujeres hemos adoptado estos papeles consciente o inconscientemente. La historia de la educación de las mujeres muestra cómo a lo largo del tiempo estas han ocupado un lugar secundario y subordinado (Subirats, 1994).

La ordenación educativa sexista determina la internalización de normas de género diferenciadas (Subirats y Brullet, 1999; Tomé, 1999); ha despreciado la identidad femenina (Tomé, 1999); ha provocado que el profesorado se dirija a ellos y ellas como tales, les ponga a competir y les evalúe y sitúe en lo que equivale a una educación no mixta dentro de una educación mixta (Acker, 2003); ha producido libros de texto que ignoran la presencia de las mujeres o la han estereotipado en ciertas funciones (Acker, 2003); ha provocado que el espacio escolar haya sido divido en territorios para cada uno de los sexos (Subirats, 1999; Tomé, 1999; Acker, 2003); ha traído como consecuencia que a los hombres se les preste mayor atención en las clases y que los contenidos y trabajo en el aula se planifiquen pensando en ellos (Tomé, 1999; Acker, 2003); ha enseñado a los hombres a manejar el poder y a orientar la actividad científica (Graña, 2008).

La discriminación de las mujeres se ha presentado en diversos aspectos: se les ha excluido del reconocimiento sobre los aportes culturales y científicos realizados a la humanidad (Subirats, 1994; Fioretti, Tejero y Díaz, 2002); en el tipo de investigación realizada (Acker, 2003); en el lenguaje utilizado (Subirats, 1994; Acker, 2003); en la tipificación de los "papeles" femeninos que distintos programas de televisión y medios de comunicación proyectan (Belmonte y Guillamón, 2008); en la división del trabajo y tareas docentes (Acker, 2003); entre otros.

Aunque la responsabilidad del cambio de esta estructura no es solamente un trabajo de las instituciones educativas y del profesorado, no se puede negar el deber social que tienen las y los docentes y todas las personas relacionadas con la educación. Solamente cuando ello sea posible y se logre establecer que la problemática como tal es real, las iniciativas de cambio tendrán efecto. 
Resulta interesante observar cómo en el currículo oculto se determinan y transmiten, aún sin darnos cuenta, las características socialmente "permitidas para los hombres y mujeres". Tanto el profesorado como los y las estudiantes, en su mayoría, no están conscientes de esta situación, por lo que la desigualdad, el trato distinto, los comportamientos y actitudes "opuestas" se han "normalizado" como "reglas" del sistema educativo.

Resulta importante, desde esta perspectiva, investigar temas como:

1. ¿En qué medida los libros de texto, en todos los niveles educativos, contribuyen a estereotipar los géneros?

2. ¿Qué prácticas educativas en el aula contribuyen a reproducir la desigualdad y las relaciones de poder?

3. ¿Existe en la educación costarricense una política real que contemple la igualdad de géneros?

4. ¿Qué formas de resistencia, consciente o inconscientemente, presentan el profesorado y los y las estudiantes ante una política de igualdad de género?

5. ¿En qué medida la educación contribuye formar el hombre "duro", violento?

6. ¿Existe un nivel educativo en donde el sexismo es aún más evidente? Si fuera así, ¿a qué se debe esta situación?

7. ¿Qué contenidos del currículo escolar contribuyen a la permanencia del sexismo?

8. ¿Qué tipo de reforma, basada en contenidos, se debe realizar para ayudar a erradicación del sexismo en la educación?

9. ¿Cuáles son los principales logros que ha tenido la coeducación? ¿Qué medidas se deben llevar a cabo para garantizar el logro de los objetivos de este modelo de escuela?

Surge, entonces, la necesidad de realizar investigación orientada a analizar la interacción en el aula y la forma de "entender" el currículo escolar desde otra perspectiva.

Aunque se haya legislado a favor de la equidad de género, en el uso del lenguaje inclusivo, en las cuotas de participación en organismos políticos, en la representación para diferentes instituciones, la creación de entes encargados de velar por un trato igualitario entre hombres y mujeres, aún resulta insuficiente, pues la igualdad de género requiere un proceso de aculturación que cambie concepciones y prácticas socialmente aprendidas.

Evidentemente, si se requiere un cambio orientado hacia la igualdad de géneros la práctica educativa debe reformarse y es responsabilidad de todos dar pasos, aunque en 
principio pequeños, para lograrlo. Se deben plantear estrategias que incluyan a todos los involucrados en la educación, donde, mediante un trabajo colaborativo, se puedan eliminar las diferencias que se han construido entre ambos sexos y permitan desarrollar una educación sin discriminación.

\section{Referencias}

Acker, Sandra. (2003). Género y educación. Reflexiones sociológicas sobre mujeres, enseñanza y feminismo. Madrid, España: Narcea, S.A. de Ediciones.

Araya, Sandra. (2004). Hacia una educación no sexista. Recuperado de http://redalyc.uaemex.mx/pdf/447/44740217.pdf

Belmonte, Jorge y Guillamón, Silvia. (2008). Co-educar la mirada contra los estereotipos de género en TV. Revista Científica de Educomunicación, 31(XVI), 115-120.

Fioretti, Susana; Tejero, Graciela y Díaz, Paula. (2002). El género: ¿un enfoque ausente en la formación docente? Recuperado de http://www.biblioteca.unlpam.edu.ar/pubpdf/aljaba/n07a08fioretti.pdf

González, Rosa. (2003). Diferencias de género en el desempeño matemático de estudiantes de secundaria. Recuperado de http://redalyc.uaemex.mx/pdf/405/40515206.pdf

Graña, François. (2008). El asalto de las mujeres a las carreras universitarias "masculinas": cambio y continuidad en la discriminación de género. PRAXIS, 12, 77-86.

Salomone, Rosemary. (2007). Igualdad y diferencia. La cuestión de la equidad de género en la educación. Revista Española de Pedagogía, LXV(238), 433-446.

Subirats, Marina y Brullet, Cristina. (1999). Rosa y azul. La trasmisión de los géneros en la escuela mixta. En Marisa Belausteguigoitia y Araceli Mingo (Eds.) Géneros Prófugos. Feminismo y educación (pp.189-223). México: Editorial Paidós Mexicana, S.A.

Subirats, Marina. (1994). Conquistar la igualdad: la coeducación hoy. Recuperado de http://www.rieoei.org/oeivirt/rie06a02.htm

Subirats, Marina. (1999). Género y escuela. En Carlos Lomas (Comp.) ¿lguales o diferentes? Género, diferencia sexual, lenguaje y educación (pp. 19-31). España: Ediciones Paidós Ibérica, S.A.

Tomé, Amparo. (1999). Un camino hacia la coeducación (instrumentos de reflexión e intervención). En Carlos Lomas (Comp.) ¿lguales o diferentes? Género, diferencia sexual, lenguaje y educación (pp. 171-197). España: Ediciones Paidós lbérica, S.A. 